Review Article

\title{
Oral Huangqi Formulae for Stable Chronic Obstructive Pulmonary Disease: A Systematic Review and Meta-Analysis
}

\author{
Lei Wu, ${ }^{1}$ Yuanbin Chen, ${ }^{1}$ Yinji Xu, ${ }^{1}$ Xinfeng Guo, ${ }^{1}$ Xiaoyan Li, ${ }^{1}$ Anthony Lin Zhang, \\ Brian H. May, ${ }^{2}$ Charlie Changli Xue, ${ }^{1,2}$ Zehuai Wen, ${ }^{1,3}$ and Lin Lin ${ }^{1}$ \\ ${ }^{1}$ Guangdong Provincial Hospital of Chinese Medicine, Guangzhou 510120, China \\ ${ }^{2}$ Discipline of Chinese Medicine, School of Health Sciences, RMIT University, Bundoora, VIC 3083, Australia \\ ${ }^{3}$ National Center for Design Measurement and Evaluation in Clinical Research, Guangzhou University of Chinese Medicine, \\ Guangzhou 510405, China
}

Correspondence should be addressed to Zehuai Wen; wenzh@gzucm.edu.cn and Lin Lin; drlinlin620@163.com

Received 1 September 2012; Revised 29 November 2012; Accepted 19 January 2013

Academic Editor: Taixiang Wu

Copyright (C) 2013 Lei Wu et al. This is an open access article distributed under the Creative Commons Attribution License, which permits unrestricted use, distribution, and reproduction in any medium, provided the original work is properly cited.

\begin{abstract}
Objective. To evaluate the efficacy and safety of oral Huangqi formulae for the treatment of stable COPD. Methods. The major databases were searched until September 2010 and supplemented with a manual search. Randomized controlled trials (RCTs) of oral Huangqi formulae that reported on lung function, St. George's Respiratory Questionnaire, symptom improvement and/or frequency of exacerbations were extracted by two reviewers. The Cochrane tool was used for the assessment of risk of bias in the included trials. Data were analyzed with RevMan 5.1.2 software. Results. 25 RCTs (1,661 participants) were included. Compared with conventional therapy (CT) alone, oral Huangqi formulae plus CT increased $\mathrm{FEV}_{1}$, and a similar result was found comparing Huangqi formulae with no treatment. Improvements in SGRQ total score, COPD-related symptoms and reduction of frequency of exacerbations were found in patients receiving Huangqi formulae plus CT compared to those receiving CT alone or CT plus placebo. No serious adverse events were reported. However, there were some methodological inadequacies in the included studies. Conclusions. The benefits of Huangqi formulae for stable COPD were promising, but its efficacy and safety have not been established due to methodological weakness and possible bias in the reported results. Further rigorously designed studies are warranted.
\end{abstract}

\section{Introduction}

Chronic obstructive pulmonary disease (COPD) is characterized by airflow limitation and manifests as progressive dyspnea accompanied by deterioration of lung function [1]. It is a major cause of morbidity, disability, and mortality. The World Health Organization (WHO) estimated that COPD ranked fifth in terms of burden of disease worldwide [2]. In China, COPD affects $8.2 \%$ of people aged 40 years or older [3]. It was the fourth leading cause of death in cities and the third in rural areas [4].

Studies have shown that pharmacotherapy cannot modify the trend of decline in lung function $[5,6]$. Chinese herbal medicine is commonly used for COPD, especially for the stable stage, in China and other Asian countries. Clinical studies suggest that herbal formulae that include Huangqi
(Radix Astragalus membranaceus) were effective for stable COPD [7-31]. It often serves as a principal medicine (i.e., the ingredient provides the principal curative action on the main syndrome or primary symptom [32]) in formulae for COPD. However, the quality of these studies had not been evaluated systematically, and some of the reports for the effects of Huangqi were conflicting. Therefore, this systematic review was conducted to evaluate the evidence for the efficacy and safety of oral Huangqi for treating stable COPD.

\section{Methods}

2.1. Search Strategy. Comprehensive searches were performed for three English language databases and five Chinese databases, which included PubMed (from 1966), Embase 
(from 1985), Cochrane Central Register of Controlled Trials, Chinese Biomedical Database (CBM, from 1979), China National Knowledge Infrastructure (CNKI, from 1994), VIP medicine information system (VMIS, from 1989), Wanfang (from 1998), and TCM-Online (from 1949), from the inceptions of the databases to September 2010, without language restriction. A manual search was conducted of evidencebased medicine (EBM) reports on Chinese prescriptions by Japan Society for Oriental Medicine, EBM special committee [31]. The focus of the search was randomized controlled trials (RCTs) of oral Huangqi formula for stable COPD.

Search terms included chronic obstructive pulmonary disease, chronic bronchitis, emphysema, COPD, chronic obstructive lung disease, chronic obstructive airway disease, chronic airflow obstruction, traditional Chinese medicine, Chinese herbal drugs, complementary and alternative medicine, phytotherapy, herbs, herbal Medicine, Astragalus, Huangqi, Beiqi, Milkvetch Root, controlled clinical trial, and their synonyms. The literature was screened based on title, abstract, and full text as needed. Full details on the search strategy are described in the appendix.

2.2. Study Selection. The inclusion criteria were as follows: (1) RCTs with patients diagnosed with COPD in the stable stage $[1,33]$, which manifests as dyspnea, cough, and phlegm which remain stable or are rather mild; (2) Huangqi formula (taken orally as decoction, pill, powder, or capsule) alone or in combination with conventional therapy compared with placebo, no treatment, or conventional therapy as controls. Huangqi serves as a principal medicine, defined as follows: the properties of Huangqi are consistent with the main aims of the formula, or the dosage of Huangqi is relatively large (more than $15 \mathrm{~g}$ ). Conventional therapy includes bronchodilators (beta2-agonists, anticholinergics, methylxanthine), corticosteroids, exercise training, smoking cessation, etc [1]. (3) Outcome measurements include spirometric parameters (forced expiratory volume in one second, $\mathrm{FEV}_{1}$ ), St. George's Respiratory Questionnaire (SGRQ) total score, symptom improvement, and/or frequency of exacerbations. The exclusion criteria were (1) trials that included patients with asthma or other non-COPD disorders; (2) test interventions that were combined with other TCM therapies such as acupuncture, acupoint injection; (3) Chinese herbs or other TCM therapies were used in the control group.

2.3. Data Extraction and Risk of Bias Assessment. Two authors (Lei Wu and Yuanbin Chen) independently assessed studies based on the inclusion and exclusion criteria. If needed, two other reviewers (Lin Lin and Zehuai Wen) were consulted. Data on the details of study design, participants, interventions, control medicine, outcome measures, numbers of dropouts, and number and nature of any adverse events reported were extracted to a predefined form.

Assessment of symptom improvement was based on the chronic bronchitis section on the Guidance for Clinical Research on New Drugs of TCM [34], where responses were categorized into four levels (symptom control, very good, good, and no effect). The proportion of patient responses for the following symptoms (cough, sputum, dyspnea, and rale) was assessed according to the previous levels.

Two authors (Lei Wu and Yuanbin Chen) independently assessed the risk of bias of the included studies using the Cochrane tool [35]. Any discrepancies in assessment were decided by discussion. Other authors (Zehuai Wen and Xinfeng Guo) were consulted to make the final decision when needed. To verify unclear information on methodology and therapy, attempts were made to contact the authors of the original papers via phone, email, or mail. If the authors were not contactable after 3 times by phone or email, they were sent mail and given one month to reply.

2.4. Data Analysis. Data were analyzed by RevMan 5.1.2 (Cochrane Collaboration), and Stata 12.0 software (StataCorp LP, College Station, TX, USA). Dichotomous data were presented as risk ratio (RR) and continuous outcomes as mean difference (MD), with 95\% confidence intervals $(95 \%$ CI). Statistical heterogeneity was assessed by Cochrane's $Q$ test. If the analysis showed low heterogeneity $(P \geq 0.10$ and $I^{2} \leq 50 \%$ ), data were synthesized using a fixed-effects model. Otherwise, a random-effects model was applied. Subgroup analyses were performed if sufficient numbers of RCTs were available and sensitivity analyses were undertaken as required. Publication bias was assessed by funnel plot analysis if the group included more than five trials [36]. Egger's test was conducted if it was difficult to determine whether the funnel plots were symmetrical.

\section{Results}

3.1. Description of Studies. Of 13,254 potentially relevant citations, 8,952 were excluded as duplications, 4,012 were excluded for not meeting the inclusion criteria after reading the titles and abstracts, and further 265 studies were excluded after reading the full articles and/or contacting the authors. Finally, 25 studies (including 1,661 participants) that met all the selection criteria were included (Figure 1).

Of these 25 studies, 24 were conducted in China [730] and 1 in Japan [31]. Twenty-two were published and 3 were dissertations $[20,22,26]$. All patients were diagnosed as stable COPD according to the Global Initiative for Chronic Obstructive Lung Disease (GOLD) or guidelines issued by Committee of Respiratory Disease, Chinese Medical Association. Disease severity in the included trials ranged from mild to very severe COPD. The duration of patients' COPD ranged from 4 to 30 years.

Nineteen studies compared Huangqi formulae plus conventional therapy with conventional therapy [7, 9-11, 1419, 22, 24-31]. Two studies compared Huangqi formulae plus conventional therapy with placebo plus conventional therapy $[12,21]$. Three studies compared Huangqi formulae with no treatment $[8,13,23]$. One study compared a Huangqi formula with conventional therapy [20]. The duration of treatment varied from 2 weeks to 6 months. One trial reported a 1-year follow-up period [16]. 


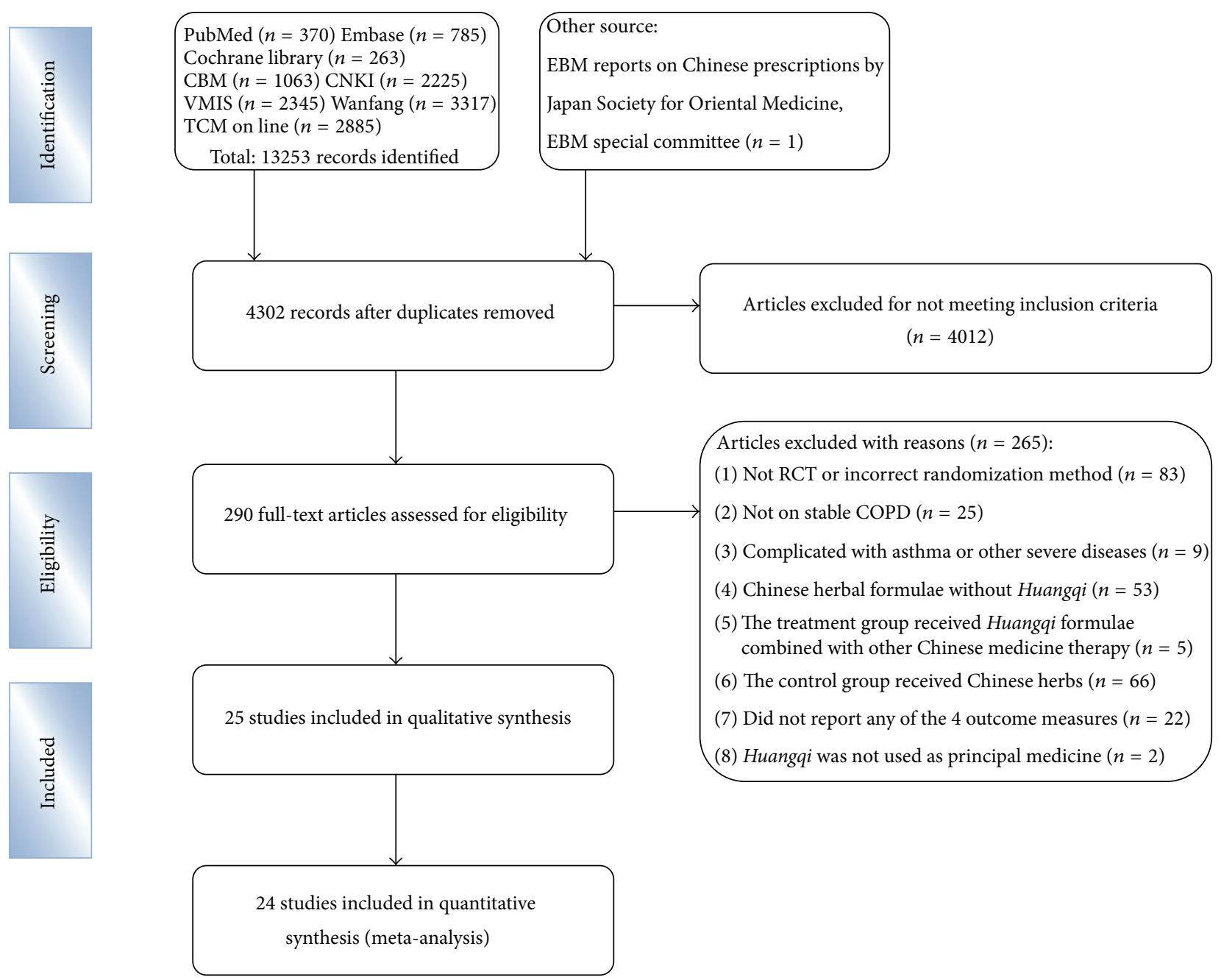

FIGURE 1: Flow diagram showing the trial selection process for the systematic review.

Five studies indicated that Huangqi was used as the principal medicine $[12,17,18,20,26]$. In the twenty remaining studies, we considered Huangqi to be the principal medicinal when its properties were consistent with the main aims of the formulae or its dosage was more than $15 \mathrm{~g}$, even when the authors did not indicate that it was used as a principal medicinal. The characteristics of the included studies are summarized in Tables 1 and 2.

3.2. Risk of Bias of the Included Studies. After attempts at verification by contacting the authors of the original papers through phone, e-mail, or mail, 14 of the included trials still lacked a detailed description of the method of randomization, and 19 trials did not indicate whether blinding was applied. Only two trials described the method of generation of randomization sequence, allocation concealment, double blinding, and placebo manufacturing method. Two trials reported dropouts; one of them explained the reason was loss of contact with the participants, and the other did not provide reasons. No trials reported whether they had used intention-to-treat analysis. Selective outcome reporting was judged as low risk of bias in 2 trials, uncertain risk in 19 trials, and high risk in 4 trials. No study had a registered or published protocol; so, judgment was based on the outcome measures specified in the method section of the study and was informed by discussion with the author when possible. Most of the included trials lacked a sample size calculation (Figure 2).

3.3. Publication Bias. The number of studies reporting SGRQ scores and the frequency of exacerbations was less than five; so, a funnel plot was not applicable. The characteristics of studies reporting $\mathrm{FEV}_{1}$ were different, and the number of trials in each subgroup was less than five; so, funnel plots were also not applicable. For symptom improvement, the symmetry of the funnel plot was not clear; so, Egger's test was conducted. It indicated that the effect of publication bias was not significant $(t=1.39,95 \% \mathrm{CI},-0.69$ to $2.48, P=0.215$ ) (Figure 3). 
TABLE 1: Characteristics of included studies.

\begin{tabular}{|c|c|c|c|c|c|}
\hline $\begin{array}{l}\text { First author, } \\
\text { year [Ref] }\end{array}$ & Location & $\begin{array}{l}\text { No. of } \\
\text { participants } \\
\text { (R/A) }\end{array}$ & $\begin{array}{l}\text { Age } \\
\text { Mean } \pm \text { SD } \\
\text { (years) }\end{array}$ & Severity of COPD & $\begin{array}{l}\text { COPD History } \\
\text { Mean } \pm \text { SD } \\
\text { (years) }\end{array}$ \\
\hline $\begin{array}{l}\mathrm{Xu} \\
2007[7]\end{array}$ & China & $\begin{array}{l}\mathrm{T}: 30 / 30 \\
\mathrm{C}: 30 / 30\end{array}$ & $\begin{array}{l}\mathrm{T}: 67 \pm 19.31 \\
\mathrm{C}: 64 \pm 20.97\end{array}$ & $\begin{array}{l}\text { T: II: } 12, \text { III: } 18 \\
\text { C: II: } 13, \text { III: } 17\end{array}$ & NR \\
\hline $\begin{array}{l}\text { Hong } \\
2004[8]\end{array}$ & China & $\begin{array}{l}\mathrm{T}: 20 / 20 \\
\mathrm{C}: 18 / 18\end{array}$ & $\begin{array}{l}\mathrm{T}: 67.70 \pm 5.68 \\
\mathrm{C}: 66.89 \pm 5.57\end{array}$ & $\begin{array}{l}\text { T: II: } 5 \text {, III: } 15 \\
\text { C: II: } 4 \text {, III: } 14\end{array}$ & $\begin{array}{l}\text { T: } 15.05 \pm 5.54 \\
\text { C: } 14.33 \pm 7.28\end{array}$ \\
\hline $\begin{array}{l}\text { Jia } \\
2007[9]\end{array}$ & China & $\begin{array}{l}\mathrm{T}: 30 / 30 \\
\mathrm{C}: 25 / 25\end{array}$ & $\begin{array}{l}\mathrm{T}: 61.6 \pm 6.1 \\
\mathrm{C}: 63.2 \pm 5.3\end{array}$ & $\begin{array}{l}\text { T: I: 5, II: 18, III: } 7 \\
\text { C: I: 3, II: 17, III: } 5\end{array}$ & $\begin{array}{l}\mathrm{T}: 15.25 \pm 4.01 \\
\mathrm{C}: 13.12 \pm 3.38\end{array}$ \\
\hline $\begin{array}{l}\text { Wang } \\
2005[10]\end{array}$ & China & $\begin{array}{l}\text { T: } 20 / 20 \\
\text { C: } 20 / 20\end{array}$ & $\begin{array}{l}\mathrm{T}: 59.4 \pm 7.5 \\
\mathrm{C}: 60.9 \pm 7.9\end{array}$ & $\begin{array}{l}\text { T: II A: } 8 \text {, II B: 9, III: } 3 \\
\text { C: II A: 9, II B: 9, III: } 2\end{array}$ & $\begin{array}{l}\mathrm{T}: 11.2 \pm 4.1 \\
\mathrm{C}: 11.8 \pm 4.5\end{array}$ \\
\hline $\begin{array}{l}\text { Cui } \\
2004[11]\end{array}$ & China & $\begin{array}{l}\text { T: } 20 / 20 \\
\text { C: } 20 / 20\end{array}$ & $\begin{array}{l}\mathrm{T}: 62.5 \\
\mathrm{C}: 61.5\end{array}$ & NR & NR \\
\hline $\begin{array}{l}\text { Lin } \\
2003[12]\end{array}$ & China & $\begin{array}{l}\mathrm{T}: 30 / 30 \\
\mathrm{C}: 30 / 30\end{array}$ & $\begin{array}{l}\text { T: } 62 \\
\text { C: } 60.5\end{array}$ & NR & $\begin{array}{l}\mathrm{T}: 16 \pm \mathrm{NR} \\
\mathrm{C}: 15.4 \pm \mathrm{NR}\end{array}$ \\
\hline $\begin{array}{l}\text { Zhang } \\
2006[13]\end{array}$ & China & $\begin{array}{l}\mathrm{T}: 24 / 24 \\
\mathrm{C}: 22 / 22\end{array}$ & $\begin{array}{l}\mathrm{T}: 66.70 \pm 6.60 \\
\mathrm{C}: 67.89 \pm 4.57\end{array}$ & $\begin{array}{l}\text { T: II: 5, III: } 19 \\
\text { C: II: } 4 \text {, III: } 18\end{array}$ & $\begin{array}{l}\mathrm{T}: 14.05 \pm 6.54 \\
\mathrm{C}: 15.33 \pm 5.0\end{array}$ \\
\hline $\begin{array}{l}\text { Feng } \\
2006[14]\end{array}$ & China & $\begin{array}{l}\mathrm{T}: 36 / 36 \\
\mathrm{C}: 33 / 33\end{array}$ & $48-73$ & NR & NR \\
\hline $\begin{array}{l}\text { Zhao } \\
2003 \text { [15] }\end{array}$ & China & $\begin{array}{l}\mathrm{T}: 15 / 15 \\
\mathrm{C}: 15 / 15\end{array}$ & $\begin{array}{l}\mathrm{T}: 51.71 \pm 8.10 \\
\mathrm{C}: 48.87 \pm 7.73\end{array}$ & NR & NR \\
\hline $\begin{array}{l}\mathrm{Li} \\
2006[16]\end{array}$ & China & $\begin{array}{l}\text { T: } 85 / 85 \\
\text { C: } 40 / 40\end{array}$ & $\begin{array}{l}\mathrm{T}: 53 \\
\mathrm{C}: 49\end{array}$ & NR & $\begin{array}{l}\mathrm{T}: 22 \pm \mathrm{NR} \\
\mathrm{C}: 25 \pm \mathrm{NR}\end{array}$ \\
\hline $\begin{array}{l}\mathrm{Xu} \\
2004[17]\end{array}$ & China & $\begin{array}{l}\mathrm{T}: 25 / 25 \\
\mathrm{C}: 24 / 24\end{array}$ & $\begin{array}{l}\mathrm{T}: 63 \pm 6.4 \\
\mathrm{C}: 62.9 \pm 7.0\end{array}$ & NR & $\begin{array}{l}\mathrm{T}: 17.7 \pm 9.5 \\
\mathrm{C}: 16.9 \pm 12.3\end{array}$ \\
\hline $\begin{array}{l}\text { Luo } \\
2002[18]\end{array}$ & China & $\begin{array}{l}\mathrm{T}: 36 / 36 \\
\mathrm{C}: 20 / 20\end{array}$ & $\begin{array}{l}\mathrm{T}: 63.86 \pm 12.56 \\
\mathrm{C}: 63.90 \pm 12.56\end{array}$ & NR & $\begin{array}{l}\text { T: } 20.36 \pm 11.21 \\
\text { C: } 19.70 \pm 10.00\end{array}$ \\
\hline $\begin{array}{l}\text { Wang } \\
2007[19]\end{array}$ & China & $\begin{array}{l}\text { T: } 30 / 30 \\
\text { C: } 30 / 30\end{array}$ & $\begin{array}{l}\mathrm{T}: 62.8 \\
\mathrm{C}: 60.5\end{array}$ & NR & $\begin{array}{l}\mathrm{T}: 15 \pm \mathrm{NR} \\
\mathrm{C}: 18 \pm \mathrm{NR}\end{array}$ \\
\hline $\begin{array}{l}\text { Huang } \\
2008[20]\end{array}$ & China & $\begin{array}{l}\text { T: } 16 / 16 \\
\text { C: } 16 / 16\end{array}$ & $\begin{array}{l}\mathrm{T}: 74.75 \pm 6.17 \\
\mathrm{C}: 73.81 \pm 3.71\end{array}$ & $\begin{array}{l}\text { T: II: } 8, \text { III: } 8 \\
\text { C: II: } 8, \text { III: } 8\end{array}$ & NR \\
\hline $\begin{array}{l}\mathrm{He} \\
2010[21]\end{array}$ & China & $\begin{array}{l}\text { T: } 49 / 49 \\
\text { C: } 49 / 49\end{array}$ & $75 \pm 5.8$ & NR & $11.25 \pm 5.86$ \\
\hline $\begin{array}{l}\text { Guan } \\
2006[22]\end{array}$ & China & $\begin{array}{l}\mathrm{T}: 37 / 30 \\
\mathrm{C}: 37 / 30\end{array}$ & $\begin{array}{l}\mathrm{T}: 65.5 \pm 8.21 \\
\mathrm{C}: 65.10 \pm 12.42\end{array}$ & $\begin{array}{l}\text { T: 0:7, I: 4, II: 4, III: } 15 \\
\text { C: 0:4, I: 5, II: } 11 \text {, III: } 10\end{array}$ & NR \\
\hline $\begin{array}{l}\text { Wei } \\
2007[23]\end{array}$ & China & $\begin{array}{l}\mathrm{T}: 60 / 60 \\
\mathrm{C}: 60 / 60\end{array}$ & Not reported & NR & NR \\
\hline $\begin{array}{l}\mathrm{Ma} \\
2010[24]\end{array}$ & China & $\begin{array}{l}\text { T: } 30 / 30 \\
\text { C: } 30 / 30\end{array}$ & Not reported & I to II & NR \\
\hline $\begin{array}{l}\text { Ding } \\
2009[25]\end{array}$ & China & $\begin{array}{l}\text { T: } 53 / 53 \\
\text { C: } 53 / 53\end{array}$ & $\begin{array}{l}\mathrm{T}: 61.23 \pm 3.65 \\
\mathrm{C}: 62.16 \pm 3.03\end{array}$ & $\begin{array}{l}\text { T: I: } 35, \mathrm{II}: 18 \\
\text { C: I: } 31, \mathrm{II}: 22\end{array}$ & $\begin{array}{l}\mathrm{T}: 17.21 \pm 3.02 \\
\mathrm{C}: 16.79 \pm 2.41\end{array}$ \\
\hline $\begin{array}{l}\text { Zhou } \\
2005[26]\end{array}$ & China & $\begin{array}{l}\mathrm{T}: 30 / 30 \\
\mathrm{C}: 30 / 30\end{array}$ & $\begin{array}{l}\mathrm{T}: 63.63 \pm 7.77 \\
\mathrm{C}: 64.83 \pm 7.37\end{array}$ & NR & NR \\
\hline $\begin{array}{l}\mathrm{Hu}, 2009 \\
{[27]}\end{array}$ & China & $\begin{array}{l}\text { T: } 35 / 35 \\
\text { C: } 32 / 32\end{array}$ & $\begin{array}{l}\mathrm{T}: 64.7 \pm 7.5 \\
\mathrm{C}: 63.2 \pm 5.4\end{array}$ & NR & NR \\
\hline $\begin{array}{l}\text { Chen } \\
2009[28]\end{array}$ & China & $\begin{array}{l}\mathrm{T}: 30 / 30 \\
\mathrm{C}: 30 / 30\end{array}$ & 70.1 & NR & NR \\
\hline $\begin{array}{l}\text { Liang } \\
2009 \text { [29] }\end{array}$ & China & $\begin{array}{l}\mathrm{T}: 32 / 32 \\
\mathrm{C}: 33 / 33\end{array}$ & $\begin{array}{l}\text { T: } 69.73 \\
\text { C: } 70.69\end{array}$ & $\begin{array}{l}\text { T: I: 0, II: 13, III: 12, IV: } 7 \\
\text { C: I: 0, II: } 15 \text {, III: } 12 \text {, IV: } 6\end{array}$ & $\begin{array}{l}\mathrm{T}: 17.32 \pm \mathrm{NR} \\
\mathrm{C}: 17.02 \pm \mathrm{NR}\end{array}$ \\
\hline $\begin{array}{l}\text { Zhang } \\
2009[30]\end{array}$ & China & $\begin{array}{l}\mathrm{T}: 60 / 56 \\
\mathrm{C}: 60 / 48\end{array}$ & 68.4 & NR & NR \\
\hline $\begin{array}{l}\text { Tatsumi } \\
2002[31]\end{array}$ & Japan & $\begin{array}{l}\text { T: } 34 / 34 \\
\text { C: } 37 / 37\end{array}$ & Not reported & II to III & NR \\
\hline
\end{tabular}

T: treatment; C: control; NR: not reported; R: number of subjects randomized; A: number of subjects analysed; SD: standard deviation. 


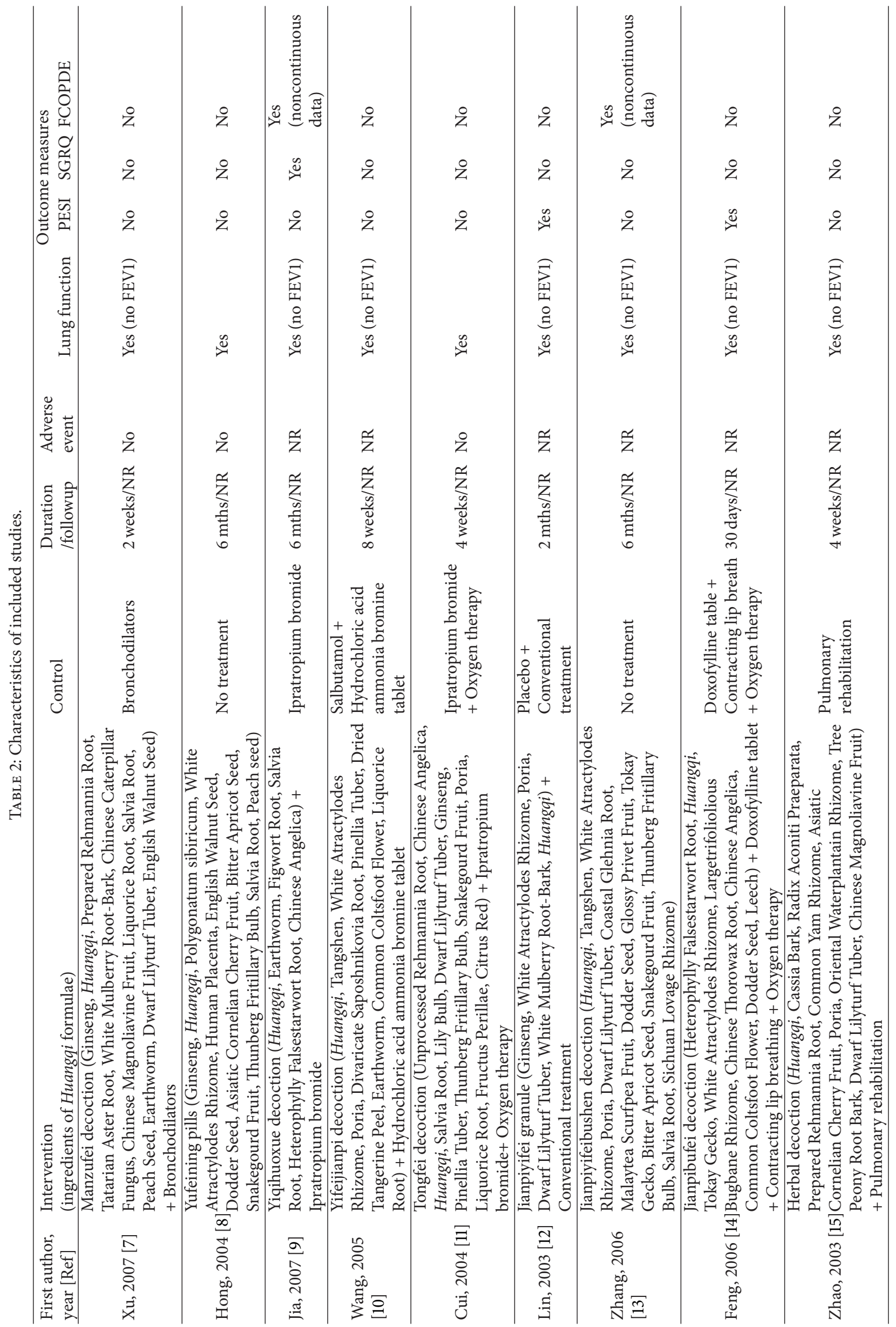




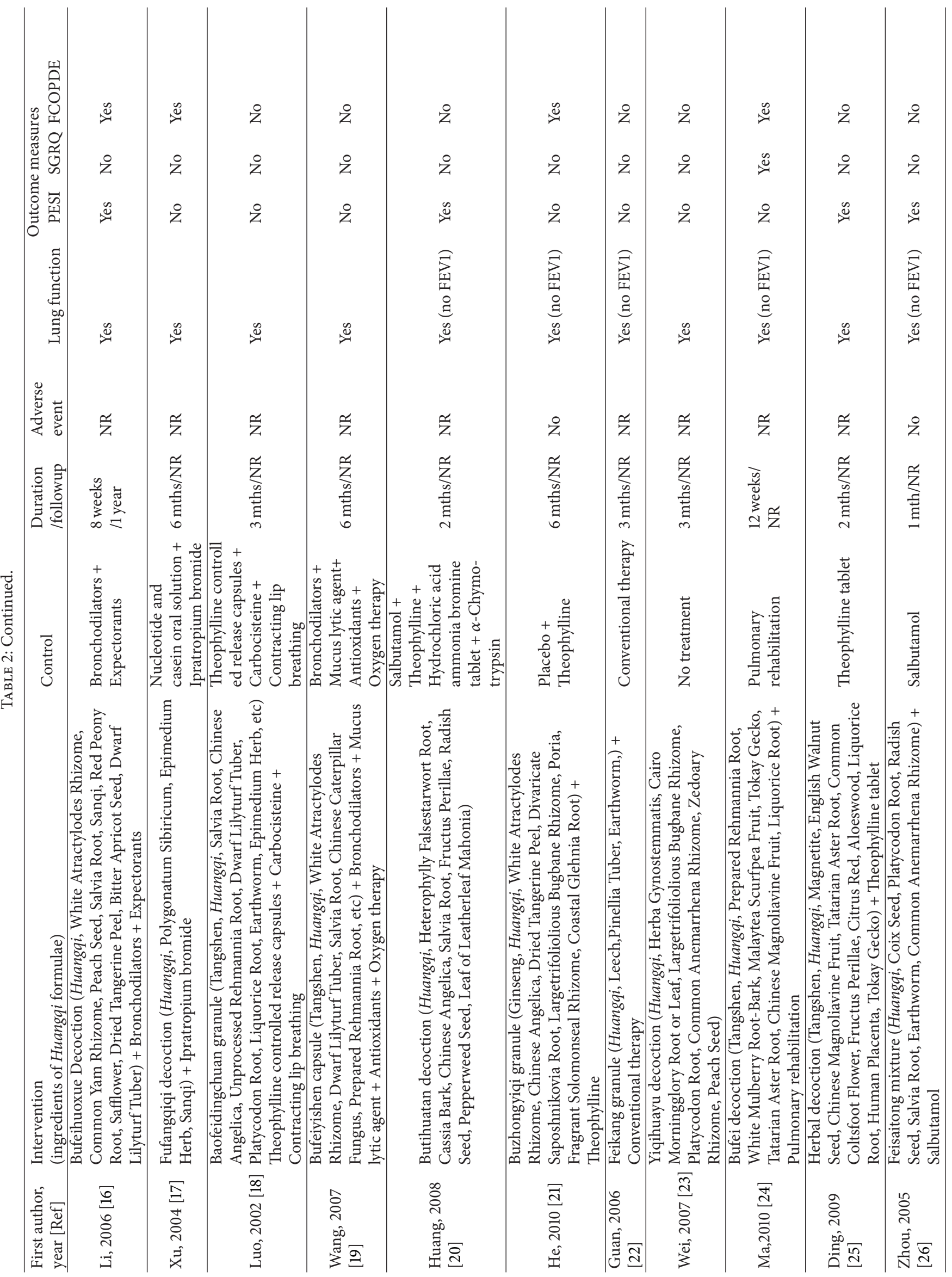




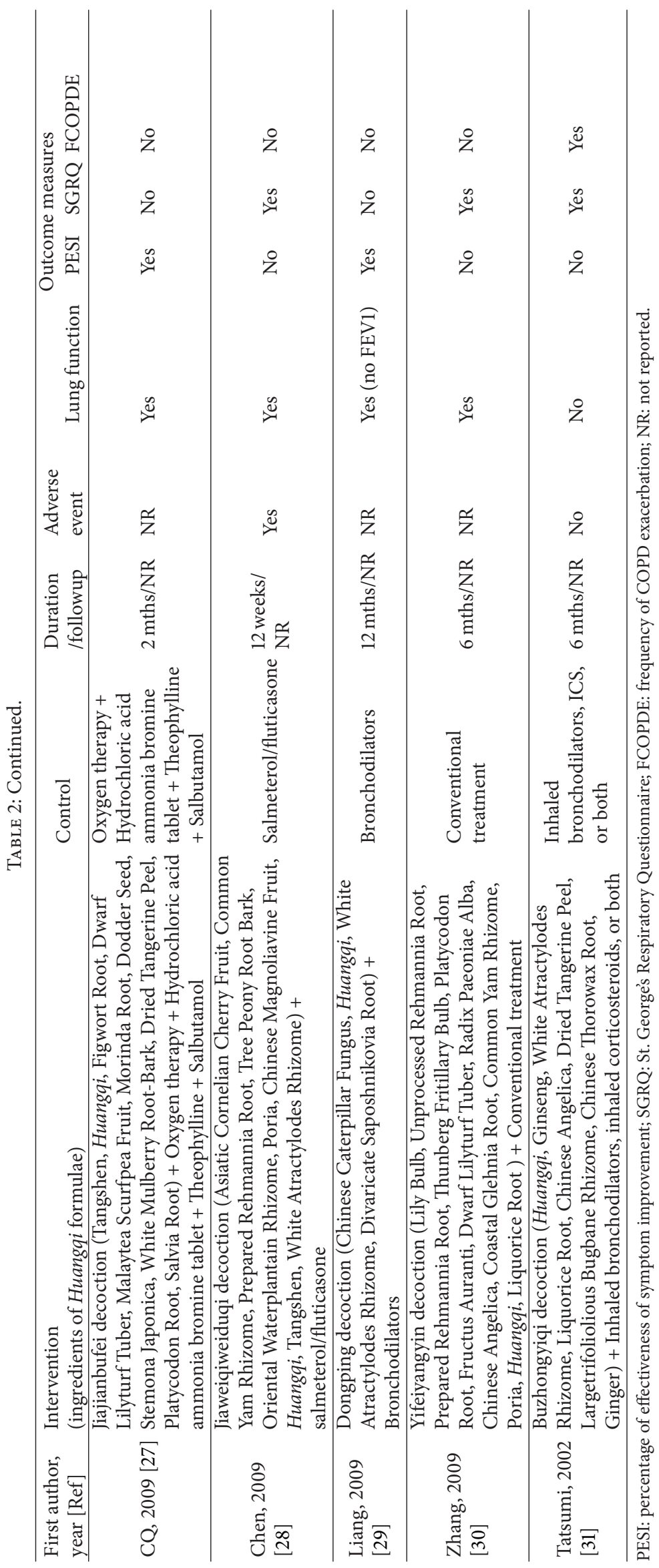




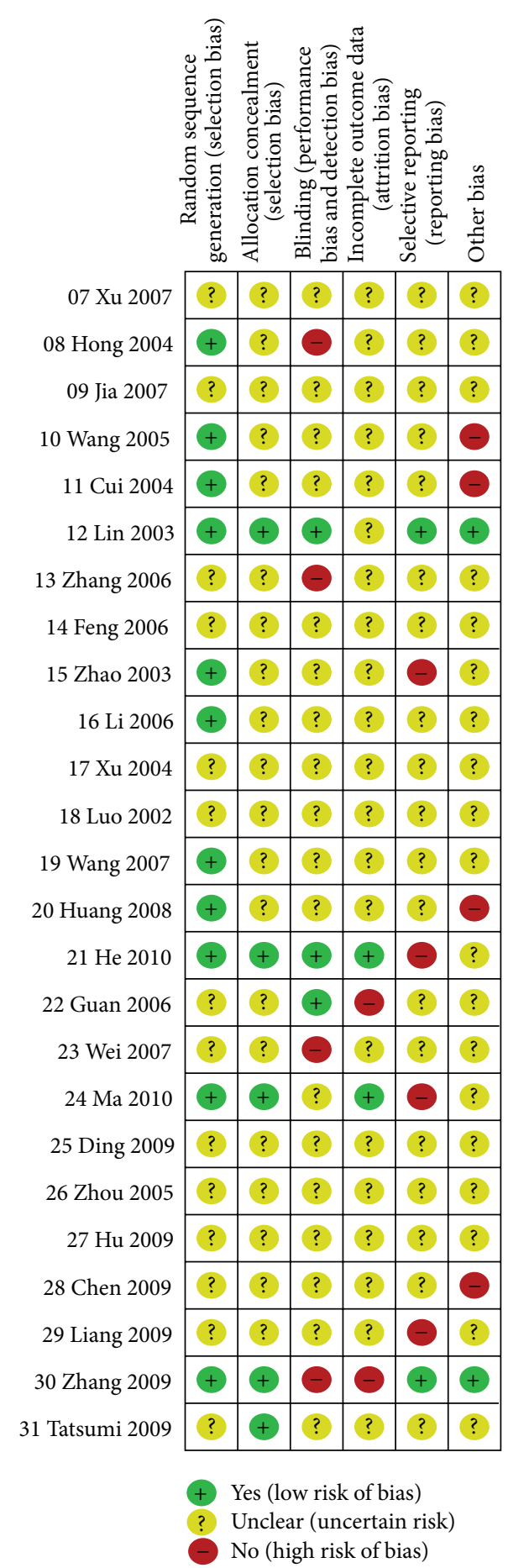

FIGURE 2: Summary of assessment of risk of bias for each included study.

\subsection{Outcome Measures}

3.4.1. Spirometric Parameters. Subgroup analyses were performed according the type of comparison (Figure 4). Subgroup 1 compared Huangqi formulae plus conventional therapy with conventional therapy alone $[11,16-19,25,27,28,30]$. $\mathrm{FEV}_{1}$ increased $0.27 \mathrm{~L}$ (95\% CI: 0.11 to 0.43 ) based on 9

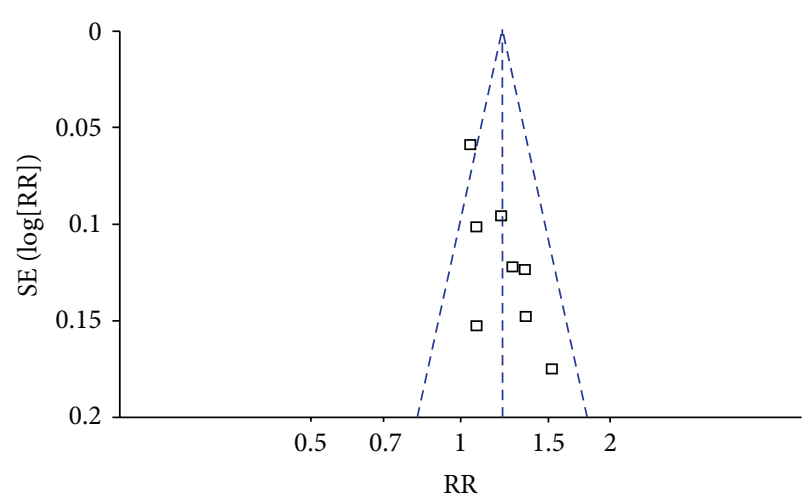

FIGURE 3: Funnel plot of publication bias using symptom improvement.

trials, but there was high heterogeneity in this subgroup. So, further analyses were performed according to duration of treatment as follows: more than, equal to, or less than 3 months. The former 2 categories had low heterogeneity. There was a significant difference in $\mathrm{FEV}_{1}$ in the trials which had durations of more than 3 months (MD 0.33, 95\% CI: 0.20 to 0.46$)[17,19,30]$ and no significant difference in those of 3 months duration (MD $0.07,95 \% \mathrm{CI}:-0.07$ to 0.20 ) $[18,28]$. For trials of less than 3 months [11, 16, 25, 27], $\mathrm{FEV}_{1}$ increased (MD 0.36, 95\% CI: 0.29 to 0.43 ), but there was heterogeneity. So, sensitivity analysis was conducted. It produced low heterogeneity with a significant difference (MD $0.49,95 \%$ CI: 0.41 to 0.57 ) when one trial [16] was removed because the age distribution of participants differed from the other trials.

Subgroup 2 compared Huangqi formulae with no treatment $[8,23] . \mathrm{FEV}_{1}$ increased significantly by $0.19 \mathrm{~L}$ based on 2 trails (95\% CI: 0.10 to 0.28 ).

3.4.2. Quality of Life. Patients receiving Huangqi formulae plus conventional therapy showed a significantly greater reduction in SGRQ total score (MD $-5.04,95 \% \mathrm{CI}:-7.48$ to -2.61) than those receiving conventional therapy alone, based on 3 trials [24, 28, 30] (Figure 5).

3.4.3. Symptom Improvement. Patients receiving Huangqi formulae plus conventional therapy were more likely to show improvements in COPD-related symptoms (RR: 1.21, 95\% CI: 1.12 to 1.31 ) when compared with those receiving conventional therapy alone or conventional therapy plus placebo, based on 8 trials [12, 14, 16, 20, 25-27, 29] (Figure 6).

3.4.4. Frequency of Exacerbations. Four trials reported frequency of exacerbations at pre- and posttreatment $[16,17$, $21,24]$. Compared with placebo plus conventional therapy or conventional therapy alone, the frequency of exacerbations in patients receiving Huangqi formulae plus conventional therapy was significantly reduced (MD $-0.97,95 \% \mathrm{CI}:-1.57$ to -0.37 ), but the heterogeneity was high (Figure 7 ). This 


\begin{tabular}{|c|c|c|c|c|c|c|c|c|c|c|c|}
\hline \multirow[b]{2}{*}{ Study or subgroup } & \multicolumn{3}{|c|}{ Experimental } & \multicolumn{3}{|c|}{ Control } & \multicolumn{2}{|r|}{ Mean difference } & \multirow{2}{*}{\multicolumn{2}{|c|}{$\begin{array}{l}\text { Mean difference } \\
\text { IV, fixed, } 95 \% \mathrm{CI}\end{array}$}} & \\
\hline & Mean & SD & Total & Mean & SD & Total & Weight & IV, fixed, 95\% CI & & & \\
\hline \multicolumn{12}{|c|}{ 5.1.1 Huangqi formulae plus $\mathrm{CT}^{*}$ versus $\mathrm{CT}$, treatment duration $<3$ months } \\
\hline 11 Cui 2004 & 0.41 & 0.44 & 20 & 0.01 & 0.51 & 20 & $7.6 \%$ & $0.4[0.10,0.7]$ & & & \\
\hline $16 \operatorname{Li} 2006$ & 0.05 & 0.39 & 85 & 0.07 & 0.35 & 40 & $0 \%$ & $-0.02[-0.16,0.12]$ & & & \\
\hline 25 Ding 2009 & 0.72 & 0.12 & 53 & 0.2 & 0.33 & 53 & $73.7 \%$ & $0.52[0.43,0.61]$ & & & \\
\hline $27 \mathrm{Hu} 2009$ & 0.73 & 0.38 & 35 & 0.32 & 0.4 & 32 & $18.8 \%$ & $0.41[0.22,0.6]$ & & & \\
\hline Subtotal (95\% CI) & & & 108 & & & 105 & $100 \%$ & $0.49[0.41,0.57]$ & & & \\
\hline \multicolumn{12}{|c|}{$\begin{array}{l}\text { Heterogeneity: } \chi^{2}=1.45, d f=2(P=0.49) ; I^{2}=0 \% \\
\text { Test for overall effect: } Z=11.84(P<0.00001)\end{array}$} \\
\hline \multicolumn{12}{|c|}{ 5.1.2 Huangqi formulae plus CT versus CT, treatment duration $=3$ months } \\
\hline 18 Luo 2002 & 0.26 & 0.63 & 36 & 0.08 & 0.51 & 20 & $19.5 \%$ & $0.18[-0.12,0.48]$ & & & \\
\hline 28 Chen 2009 & 0.16 & 0.31 & 30 & 0.12 & 0.28 & 30 & $80.5 \%$ & $0.04[-0.11,0.19]$ & & & \\
\hline Subtotal (95\% CI) & & & 66 & & & 50 & $100 \%$ & $0.07[-0.07,0.2]$ & & & \\
\hline \multicolumn{12}{|c|}{$\begin{array}{l}\text { Heterogeneity: } \chi^{2}=0.66, d f=1(P=0.42) ; I^{2}=0 \% \\
\text { Test for overall effect: } Z=0.98(P=0.33)\end{array}$} \\
\hline \multicolumn{12}{|c|}{ 5.1.3 Huangqi formulae plus CT versus CT, treatment duration $>3$ months } \\
\hline 17 Xu 2004 & 0.27 & 0.59 & 25 & 0.07 & 0.61 & 24 & $14.9 \%$ & $0.2[-0.14,0.54]$ & & & \\
\hline 19 Wang 2007 & 0.49 & 0.65 & 30 & 0.12 & 0.44 & 30 & $21.4 \%$ & $0.37[0.09,0.65]$ & & & \\
\hline 30 Zhang 2009 & 0.48 & 0.4 & 56 & 0.14 & 0.44 & 48 & $63.7 \%$ & $0.34[0.18,0.5]$ & & & \\
\hline Subtotal (95\% CI) & & & 111 & & & 102 & $100 \%$ & $0.33[0.20,0.46]$ & & & \\
\hline \multicolumn{12}{|c|}{$\begin{array}{l}\text { Heterogeneity: } \chi^{2}=0.66, d f=2(P=0.72) ; I^{2}=0 \% \\
\text { Test for overall effect: } Z=4.91(P<0.00001)\end{array}$} \\
\hline \multicolumn{12}{|c|}{ 5.1.4 Huangqi formulae versus no treatment } \\
\hline 08 Hong 2004 & 0.05 & 0.34 & 20 & -0.04 & 0.33 & 18 & $17.3 \%$ & $0.09[-0.12,0.3]$ & & & \\
\hline 23 Wei 2007 & 0.22 & 0.33 & 60 & 0.01 & 0.2 & 60 & $82.7 \%$ & $0.21[0.11,0.31]$ & & & \\
\hline Subtotal (95\% CI) & & & 80 & & & 78 & $100 \%$ & $0.19[0.1,0.28]$ & & & \\
\hline \multicolumn{12}{|c|}{$\begin{array}{l}\text { Heterogeneity: } \chi^{2}=1.01, d f=1(P=0.32) ; I^{2}=1 \% \\
\text { Test for overall effect: } Z=4.18(P<0.0001)\end{array}$} \\
\hline & & & & & & & & & $-1 \quad-0.5$ & 0.5 & 1 \\
\hline & & & & & & & & & Favours control & Favours & rimental \\
\hline
\end{tabular}

FIGURE 4: Forest plot of Huangqi formulae plus conventional therapy versus conventional therapy, or Huangqi formulae versus no treatment in patients with stable COPD: change in $\mathrm{FEV}_{1}(\mathrm{~L})$.

\begin{tabular}{|c|c|c|c|c|c|c|c|c|}
\hline \multirow[b]{2}{*}{ Study or subgroup } & \multicolumn{3}{|c|}{ Experimental } & \multicolumn{2}{|c|}{ Control } & \multirow[b]{2}{*}{ Total } & \multirow{2}{*}{$\begin{array}{l}\text { Mean difference } \\
\text { IV, fixed, 95\% CI }\end{array}$} & \multirow{2}{*}{$\begin{array}{l}\text { Mean difference } \\
\text { IV, fixed, 95\% CI }\end{array}$} \\
\hline & Mean & SD & Total & Mean & SD & & & \\
\hline 24 Ma 2010 & -20.4 & 11.17 & 30 & -10.9 & 10.31 & 30 & $20.1 \%-9.5[-14.94,-4.06]$ & -- \\
\hline 28 Chen 2009 & -6.38 & 7.4 & 30 & -3.68 & 7.08 & 30 & $-2.7[-6.36,0.96]$ & - \\
\hline 30 Zhang 2009 & -6.36 & 10.83 & 56 & -0.92 & 10.35 & 48 & $35.7 \%-5.44[-9.52,-1.36]$ & $-1-$ \\
\hline Total $(95 \% \mathrm{CI})$ & & & 116 & & & 108 & $100 \%-5.04[-7.48,-2.61]$ & $\nabla$ \\
\hline \multicolumn{8}{|c|}{$\begin{array}{l}\text { Heterogeneity: } \chi^{2}=4.19, d f=2(P=0.12) ; I^{2}=52 \% \\
\text { Test for overall effect: } Z=4.06(P<0.0001)\end{array}$} & $\begin{array}{cccc}-20-10 & 0 & 10 & 20 \\
& & \\
& & & \\
\text { xperimental } & \text { Favours }\end{array}$ \\
\hline
\end{tabular}

FIGURE 5: Comparison of Huangqi formulae plus conventional therapy versus conventional therapy alone in patients with stable COPD: change in SGRQ total scores.

appeared due to variation between trials in terms of the control interventions used, duration, and trial quality. Overall, the better result appeared to be of He et al., 2010 [21].

3.5. Adverse Events. Six trials reported that no adverse events occurred $[7,8,11,21,26,31]$. One trial (60 participants) reported hoarseness in the control group treated by salmeterol/fluticasone [28], but there was no causality assessment for this adverse event. The other trials did not report adverse events; so, there was insufficient data to assess whether the combination of Huangqi formulae plus conventional therapy affected the adverse event rate.

\section{Discussion}

This systematic review includes 24 RCTs conducted in China and 1 RCT in Japan. All RCTs employed the herb Huangqi as a principal herb in the herbal formulae used in the test arms, 


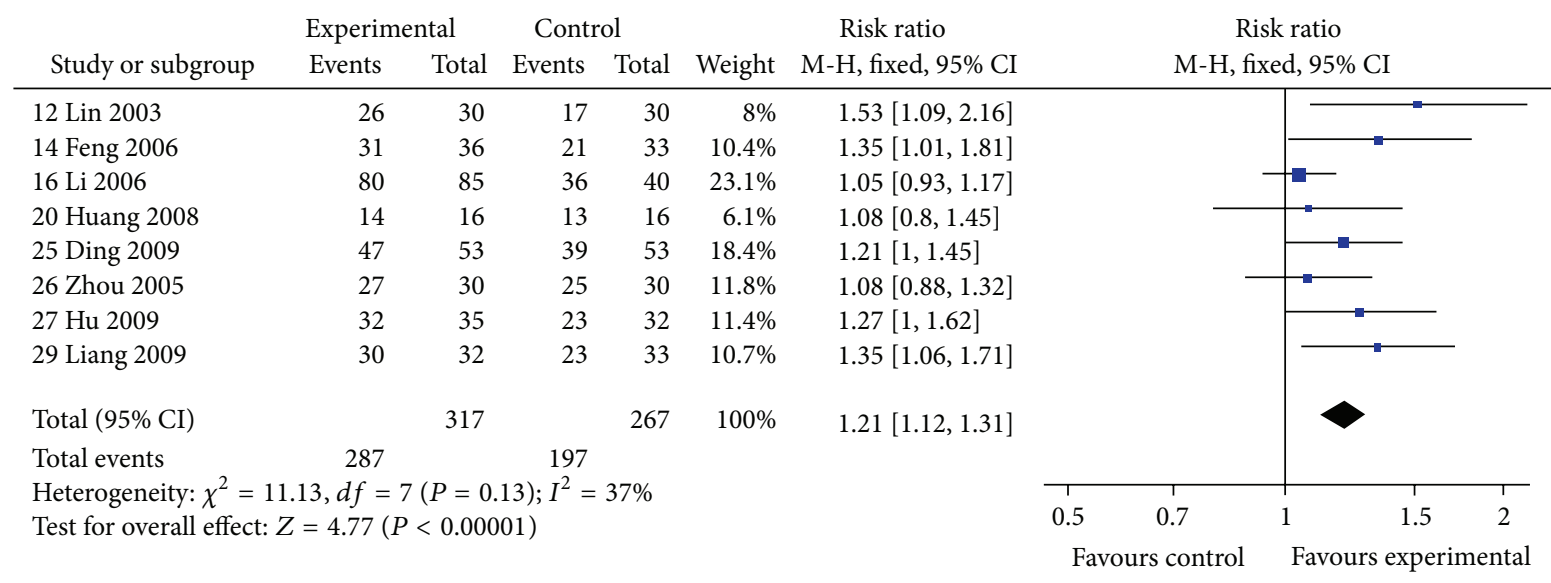

FIgure 6: Comparison of Huangqi formulae plus conventional therapy versus conventional therapy alone in patients with stable COPD: overall symptom improvement (defined as "good" or above).

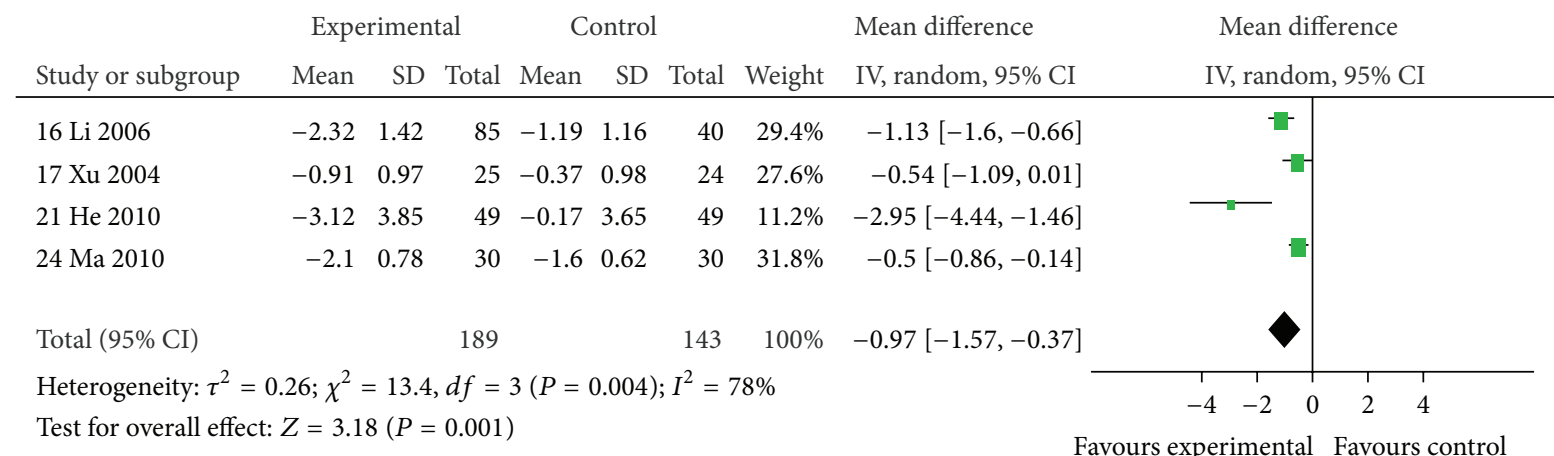

FIGURE 7: Comparison of Huangqi formulae plus conventional therapy versus placebo plus conventional therapy or conventional alone in patients with stable COPD: change in frequency of exacerbations.

and all studies only included patients assessed as suffering stable COPD. The comparators were mostly conventional therapy, but this was variable and was not clearly specified in some studies. This is likely to have been a source of heterogeneity in the meta-analyses, but it also reflects usual care since patients with stable COPD would typically receive a variety of conventional therapies which may be varied according to response and individual need. Eight studies were of six months, while the others were of shorter treatment duration and only one had a followup at one year; so, the results can only be considered relevant to the relatively shortterm management of COPD.

The meta-analysis results indicate that the use of Huangqi formulae could significantly improve lung function measured as $\mathrm{FEV}_{1}$ when compared with no treatment based on two studies, and it produced an additional improvement when combined with conventional therapies based on 9 studies, four of which were of over three months duration. The incidence of exacerbations also appeared to decline when Huangqi formulae were combined with conventional therapies. Improvements in quality of life based on SGRQ were evident, but this was based on only three studies. Also, these Huangqi-containing formulae appeared to be well tolerated, even when combined with conventional medications, since seven studies reported that no adverse events were noted.

All the included trials demonstrated at least some methodological deficiencies leading to potential risks of bias. Only eleven provided evidence of adequate randomization procedures, and only three were effectively blinded to participants and investigators. Consequently, the results should be interpreted with caution. Therefore, the potential benefits of oral Huangqi formulae for stable COPD need to be further appraised through trials that employ rigorous methodology and include adequate assessment of the safety profiles of the interventions. In addition, we found that the reporting of trial methods and procedures was frequently unclear and insufficient. Therefore, we suggest that all reports of RCTs published in China should be required to comply with the CONSORT statement [37] and the publication of protocols should be encouraged.

There has been increasing interest in complementary and alternative medicine (CAM) for the treatment of COPD, especially the use of Chinese herbal medicines [38, 39]. A recent cross-sectional study in Australia suggested that nearly one in five $(17.3 \%)$ individuals with moderate to severe COPD 
had used some form of herbal preparation [39]. Therefore, reviews of the state of the evidence base are essential.

From viewpoint of TCM, patients with stable COPD usually manifest with Qi-deficiency syndrome [40]. One of the characteristics of Qi-deficiency is that the patient easily suffers from colds which commonly lead to acute exacerbations of COPD. Huangqi is one of the principal herbs used for reinforcing Qi. It has been widely used for preventing and alleviating common colds; so, its clinical use in COPD is predicated on a putative benefit in preventing colds and reducing COPD exacerbations.

From the experimental perspective, one line of research into Huangqi has focused on its effects on inflammation. The main pathological characteristic of COPD is chronic airway inflammation involving a number of proinflammatory mediators and cytokines. Also, oxidative stress is increased in COPD, which amplifies inflammation and may result in corticosteroid resistance. An invivo study suggests that Huangqi may reduce inflammatory infiltration and inhibit the inflammatory response in the airway through downregulating the expression of TNF- $\alpha$ and IL- 8 [41]. Flavonoids in Huangqi may protect the erythrocyte membrane from attack by free radicals and appear to eliminate free radicals [42]. One study has shown that airway inflammation induced by cigarette smoke was reversed by astragaloside IV (AST IV), another active constituent of Huangqi, in a dose-dependent fashion. This effect appeared due to its anti-inflammatory and antioxidant properties, including NF- $\kappa$ B inactivation [43]. Other studies suggested that AST IV possesses antiinflammatory and immune regulation activity and can be used for preventing asthma attacks [44]. The previous pharmacological properties of Huangqi may at least partially explain the clinical benefits reported by the studies included in this paper.

An earlier paper [45] evaluated the effects of a diverse range of herbal medicines in $\mathrm{COPD}$, and a subsequent paper narrowed the focus to ginseng and ginseng-containing formulae [46]. The strengths of this paper are it focuses only on formulae that share the same principal ingredient and there is experimental evidence that supports the application of this herb as a modulator of inflammation.

The main limitations to this paper are the potential sources of bias due to methodological defects and inadequacies in reporting. Although publication bias was not a major issue, the previous issues potentially lead to overreporting of positive results, selective reporting of outcome measures, and underreporting of adverse events. Also, the use of a diversity of conventional therapies as comparators makes it difficult to assess the magnitude of any effects and to interpret their clinical significance.

Therefore, the potential benefits of oral Huangqi formulae for stable COPD evident in this paper need to be further appraised through suitably powered clinical trials that employ standardized conventional therapies as comparators over a sufficient period to determine whether any effects are of sufficient clinical relevance to warrant modification to current best practice for the management of stable COPD.

\section{Conclusions}

Oral Huangqi formulae appear beneficial in terms of improving lung function, quality of life, and symptoms and in reducing the incidence of exacerbations for patients with stable COPD, but these apparent benefits require further appraisal through higher quality trials that strictly adhere to methodological principles and procedures.

\section{Appendix}

\section{Search Strategies}

\section{English Databases}

\#1: Chronic obstructive pulmonary disease OR COPD OR emphysema OR Chronic obstructive lung disease OR Chronic obstructive airway disease OR emphysema, pulmonary disease OR Airflow obstruction, Chronic OR Chronic Airflow Obstruction OR Pulmonary Emphysema

\#2: Traditional Chinese Medicine OR Chinese Traditional Medicine OR Chinese Herbal Drugs OR Chinese Drugs, Plant OR Medicine, Traditional OR Ethnomedicine OR Ethnobotany OR Medicine, Kampo OR TCM OR Alternative Medicine OR Complementary Medicine OR Phytotherapy OR Herbology OR Plants, Medicinal OR Plant Preparations OR Plant Extracts OR Plants, Medicine OR Materia Medica OR Single Prescription OR Herbs OR Chinese Medicine Herb OR Herbal Medicine

\#3: Clinical Trial OR clinical study OR biomedical research OR Controlled Trial OR Controlled study OR random control Trial OR random control study OR Multicenter Study OR random allocation OR double-blind OR single-blind OR comparative study OR evaluation study OR follow-up study OR prospective study OR research design OR control group OR placebo control OR dummy control OR blinding OR clinical research OR medical trial OR in vivo study OR case control study OR intervention study

\#4: Astragalus OR Milkvetch OR Huangqi OR Beiqi OR Milkvetch Root

\#5: \#1 AND \#2 AND \#3

\#6: \#1 AND \#3 AND \#4

\#7: \#5 OR \#6.

Chinese Databases (search by using simplified Chinese character)

\#1: Man Xing Zu Sai Xing Fei Bing (Chronic obstructive pulmonary disease) OR Man Xing Zu Sai Xing Fei Ji Bing (Chronic obstructive pulmonary disease) OR Man Zu Fei (Chronic obstructive pulmonary disease) OR Man Xing Zu Sai Xing Fei Bu Ji Bing (Chronic obstructive lung disease) OR COPD OR Zu Sai Xing Fei Ji Bing (Obstructive pulmonary disease) OR Zu Sai Xing Fei Bing (Obstructive pulmonary disease) 
OR Man Xing Zu Sai Xing Fei Qi Zhong (Chronic obstructive pulmonary emphysema) OR Zu Sai Xing Fei Qi Zhong (Obstructive pulmonary emphysema)

\#2: Zhong Yi (Traditional Chinese Medicine) OR Guo Yi (Chinese Medicine) OR Chuan Tong Yi Xue (Traditional Medicine) OR Han Fang (Kampo) OR Han Yi (Kampo Medicine) OR Dong Yi (Vietnamese traditional medicine) OR Min Zu Yi Yao (Ethnomedicine) OR Bian Zheng (Syndrome Differentiation) OR Zhi Fa (Therapeutic Method) OR Zhi Ze (Therapeutic Principle) OR Zhong Xi Yi (Chinese and Western medicine) OR Zhong Xi Yi Jie He (Integration of Chinese and Western medicine) OR Zhong Xi Yao (Chinese and Western medicine) OR Zhong Yao (Chinese Medicine) OR Cao Yao (Herbal Medicine) OR Zhong Cheng Yao (Chinese patent medicine) OR Zhi Wu Yao (Phytomedicine) OR Zhen Ci (acupuncture) OR Jiu (moxibustion) OR Jing Luo (meridian/channel) OR Xue Wei (acupoint) OR Shui Zhen (Aqueous acupuncture) OR Er Xue (ear point) OR Er Zhen (ear acupuncture) OR Fang Xue (Bloodletting) OR Ci Xue (pricking blood) OR Ci Luo (collateral pricking) OR Dian Zhen (electronic acupuncture) OR Ba Guan (cupping) OR Tui Na (tuina) OR An Mo (massage) OR Mei Hua Zhen (plum-blossom needle) OR San Leng Zhen (three-edged needling) OR Mai Xian (catgut-embedding) OR Yao Yun (hot medicinal compress) OR Guan Chang (enema)

\#3: Huang Qi (Milkvetch) OR Bei Qi (Milkvetch) OR Huang Qi (variant of Chinese character, Milkvetch)

$$
\begin{aligned}
& \text { \#4: \#1 AND \#2 } \\
& \text { \#5: \#1 AND \#3 } \\
& \text { \#6: \#4 OR \#5. }
\end{aligned}
$$

\section{Abbreviations}

AST IV: Astragaloside IV

CAM: Complementary and alternative medicine

CBM: $\quad$ Chinese biomedical

CI: $\quad$ Confidence interval

CNKI: China national knowledge infrastructure

COPD: Chronic obstructive pulmonary disease

EBM: $\quad$ Evidence-based medicine

ETSE-CM: Evaluation of Traditional and Scientific Evidence of Chinese Medicine

FCOPDE: Frequency of COPD exacerbationdatabase

FEV1: $\quad$ Forced expiratory volume in 1 second

ICS: Inhaled corticosteroids

IL-8: Interleukin-8

IRN-TCM: International Research Network for Traditional and Complementary Medicine

ITT: Intention-to-treat

IV: $\quad$ Inverse variance

MD: $\quad$ Mean difference
NF- $\kappa$ B: Nuclear factor $-\kappa B$

PESI: Percentage of effectiveness of symptom improvement

RR: $\quad$ Risk ratio

RCTs: Randomized controlled trials

SGRQ: St. George's Respiratory Questionnaire

TCM: Traditional Chinese medicine

TNF- $\alpha$ : Tumor Necrosis Factor- $\alpha$

VMIS: VIP medicine information system

WHO: World Health Organization.

\section{Conflict of Interests}

The authors declare no conflict of interests.

\section{Acknowledgments}

This work was supported by the Joint Sino-Australia Project of the International Research Network for Traditional and Complementary Medicine (IRN-TCM), titled "Evaluation of Traditional and Scientific Evidence of Chinese Medicine (ETSE-CM)," and the Program of International S\&T Cooperation from the Ministry of Science and Technology, China (no. 2012DFA31760).

\section{References}

[1] GOLD, "Global strategy for the diagnosis, management and prevention of chronic obstructive pulmonary disease," 2010, http://www.goldcopd.

[2] A. D. Lopez and C. C. Murray, "The global burden of disease, 1990-2020," Nature Medicine, vol. 4, pp. 1241-1243, 1998.

[3] N. Zhong, C. Wang, W. Yao et al., "Prevalence of chronic obstructive pulmonary disease in China: a large, populationbased survey," American Journal of Respiratory and Critical Care Medicine, vol. 176, no. 8, pp. 753-760, 2007.

[4] X. Fang, X. Wang, and C. Bai, "COPD in China: the burden and importance of proper management," Chest, vol. 139, no. 4, pp. 920-929, 2011.

[5] N. R. Anthonisen, J. E. Connett, J. P. Kiley et al., "Effects of smoking intervention and the use of an inhaled anticholinergic bronchodilator on the rate of decline of FEV1: the lung health study," Journal of the American Medical Association, vol. 272, no. 19, pp. 1497-1505, 1994.

[6] J. Vestbo, T. Sørensen, P. Lange et al., "Long-term effect of inhaled budesonide in mild and moderate chronic obstructive pulmonary disease: a randomised controlled trial," Lancet, vol. 353, no. 9167, pp. 1819-1823, 1999.

[7] L. S. Xu, "The influence of combined treatment of traditional Chinese medicine and western medicine on pulmonary function with the patients of moderate and severe COPD," China and Foreign Medical Journal, vol. 5, no. 2, pp. 55-56, 2007.

[8] M. L. Hong, L. Y. Gao, S. Z. Dai et al., "The effects of Yufeining on respiratory muscle strength and central respiratory drive in patients with COPD," Chinese Journal of Information on Traditional Chinese Medicine, vol. 11, no. 11, pp. 961-963, 2004.

[9] G. L. Jia, F. J. Tong, D. Z. Yu et al., "The clinical study of Yiqihuoxue combined ipratropium bromide inhalation in the treatment of chronic obstructive pulmonary disease," Zhejiang 
Journal of Traditional Chinese Medicine, vol. 42, no. 9, pp. 510511, 2007.

[10] S. Wang, H. Y. Ji, N. Z. Zhang et al., "Effect of yifei jianpi recipe on inflammatory cells, levels of interleukin-8 and tumor necrosis factor-alpha in sputum from patients with chronic obstructive pulmonary disease," Chinese Journal of Integrated Traditional and Western Medicine, vol. 25, no. 2, pp. 111-113, 2005.

[11] Z. B. Cui, Y. D. Yuan, S. H. Liu et al., "Intervention effect of tongfei mixture on nocturnal hypoxia in patients with chronic obstructive pulmonary disease," Chinese Journal of Integrated Traditional and Western Medicine, vol. 24, no. 10, pp. 885-888, 2004.

[12] L. Lin, C. Y. Tang, and Y. J. Xu, "Clinical observation of spleenlung nourishing granule in treating respiratory muscle fatigue of stable chronic obstructive pulmonary disease," Shanghai Journal of Traditional Chinese Medicine, vol. 37, no. 11, pp. 1012, 2003.

[13] M. X. Zhang, "Clinical research on stable COPD treated by Jianpi Yifei Bushen method," Chinese Journal of Information on Traditional Chinese Medicine, vol. 13, no. 7, pp. 64-65, 2006.

[14] X. Z. Feng and H. Q. Yao, "Clinical research on Jianpi Bufei method combined with western medicine in treating stable chronic obstructive pulmonary disease in 36 cases," Qinghai Medical Journal, vol. 36, no. 9, pp. 82-83, 2006.

[15] W. Zhao, F. M. Luo, and C. Q. He, "Effects of lung rehabilitation and Bushen Chinese herbs in treating stable chronic obstructive pulmonary disease with lung function and quality of life," Modern Journal of Integrated Traditional Chinese and Western Medicine, vol. 12, no. 24, pp. 2643-2644, 2003.

[16] Y. Q. Li and C. X. Yang, "Clinical research on stable COPD treated by Bufei Huoxue method," Beijing Journal of Traditional Chinese Medicine, vol. 25, no. 11, pp. 643-645, 2006.

[17] D. S. Xu and C. Y. He, "Effect of Fufang Qiqi Decoction on prevention and treatment of chronic obstructive pulmonary diseases," Chinese Archives of Traditional Chinese Medicine, vol. 22, no. 6, pp. 991-992, 2004.

[18] X. F. Luo, X. J. Chai, Y. M. Chen et al., "Clinical research on Bufei Dingchuan Decoction in treating 36 cases of chronic obstructive pulmonary disease," Journal of Traditional Chinese Medicine, vol. 43, no. 4, pp. 268-270, 2002.

[19] Y. F. Wang, "Clinical research of Bufei Yishen capsule in treating COPD," Shandong Journal of Traditional Chinese Medicine, vol. 47, no. 13 , pp. 30-31, 2007.

[20] G. Huang, Clinical validation of Butihuatan decoction in treating lung spleen kidney qi deficiency with the patients of COPD [M.S. Dissertation], Guiyang University of TCM, 2008.

[21] Y. C. He, H. L. Chen, and R. F. Zhang, "Clinical research on quality of life of oral Buzhongyiqi in chronic obstructive pulmonary disease," Chinese Archives of Traditional Chinese Medicine, vol. 28, no. 3, pp. 506-507, 2010.

[22] Q. H. Guan, Therapy of Chinese medicine of Qixu Xueyu Tanzu syndrome of COPD and research of molecular biology index on airway remodeling [Doctor's Dissertation], Beijing University of Chinese Medicine, 2006.

[23] B. Wei, S. F. Cui, and Y. X. Zheng, "Yiqi Huayu Decoction treatment of 60 cases of respiratory muscle fatigue syndrome," Shanxi Journal of Traditional Chinese Medicine, vol. 28, no. 12, pp. 1590-1591, 2007.

[24] J. M. Ma, Z. R. Meng, and J. C. Yin, "Role of Bufei Tang in the recovery treatment of stable chronic obstructive pulmonary disease," Chinese Journal of Hospital Pharmacy, vol. 30, no. 5, pp. 404-406, 2010.

[25] Y. H. Ding and C. Y. Yuan, "Buyi Feishen Decoction treatment of stable chronic obstructive pulmonary disease," Heilongjiang Journal of Traditional Chinese Medicine, no. 5, pp. 24-25, 2009.

[26] Y. F. Zhou and Y. X. Jing, Clinical research on the effects of treating chronic obstructive pulmonary disease with Feisaitong Heji [Master's Dissertation], Shandong University of Traditional Chinese Medicine, 2005.

[27] C. Q. Hu, "Jiajian Bufei Decoction combined with western medicine treatment of 35 cases of stable chronic obstructive pulmonary disease," Journal of TCM Research, vol. 22, no. 11, pp. 24-26, 2009.

[28] Q. Chen, C. Xu, Y. Cai, and L. Ye, "Clinical research of combined treatment of traditional Chinese medicine and western medicine on chronic obstructive pulmonary disease," Journal of Fujian University of TCM, vol. 19, no. 4, pp. 12-14, 2009.

[29] A. W. Liang, M. X. Huang, L. X. Gu et al., "Effect of Dongpingtang with TCM theory "winter disease being cured in summer" on pulmonary function and quality of life in patients with chronic obstructive pulmonary disease in stable stage," Zhejiang Journal of Integrated Traditional Chinese and Western Medicine, vol. 19, no. 6, pp. 333-336, 2009.

[30] B. M. Zhang, Z.X. Shen, and S.F. Peng, "Yifei Yangyin decoction on the treatment of chronic obstruction pulmonary disease," Chinese Journal of Ethnomedicine and Ethnopharmacy, vol. 18, no. 24, pp. 159-160, 2009.

[31] Japan Society for Oriental Medicine EBM special committee, "2002 median reports: evidence-based medicine (EBM) reports on Chinese prescriptions," Japanese Journal of Oriental Medicine, vol. 53, no. 5, pp. 1-80, 2002.

[32] WHO Regional Office for the Western Pacific., "WHO international standard terminologies on traditional medicine in the western pacific region," WPRO Nonserial Publication, 2007.

[33] Group of Chronic Obstructive Pulmonary Diseases. Committee of Respiratory Disease. Chinese Medical Association, "Guideline for diagnosis and treatment of chronic obstructive pulmonary disease (2007)," Chinese Journal of Tuberculosis and Respiratory Disease, vol. 30, no. 1, pp. 8-17, 2007.

[34] X. Y. Zheng, Guidance for Clinical Research on New Drugs of TCM, China Medical Science Press, 2002.

[35] "Chapter 8: Assessing risk of bias in included studies," in Cochrane Handbook for Systematic Reviews of Interventions Version 5.0.2, J. P. T. Higgins and D. G. Altman, Eds.J. P. T. Higgins and S. Green, Eds., The Cochrane Collaboration, 2008, http://handbook.cochrane.org/.

[36] Z. Wang, Y. H. Zhang, and Q. Q. Xu, "Several methods of publication bias evaluation," Chinese Journal of Health Statistics, no. 26, pp. 539-541, 2009.

[37] K. F. Schulz, D. G. Altman, and D. Moher, “CONSORT 2010 statement: updated guidelines for reporting parallel group randomized trials," Annals of Internal Medicine, vol. 152, no. 11, pp. 726-732, 2010.

[38] G. P. Currie and J. G. Douglas, "ABC of chronic obstructive pulmonary disease: Non-pharmacological management," British Medical Journal, vol. 332, no. 7554, pp. 1379-1381, 2006.

[39] J. George, L. L. Ioannides-Demos, N. M. Santamaria et al., "Use of complementary and alternative medicines by patients with chronic obstructive pulmonary disease," Medical Journal of Australia, vol. 181, no. 5, pp. 248-251, 2004. 
[40] Z. G. Liu, Z. G. Li, and B. Peng, "Plasma metabonomic studies on the stable phase chronic obstructive pulmonary disease patients of Fei-qi deficiency syndrome and the Chinese materia medica intervention," Chinese Journal of Integrated Traditional and Western Medicine, vol. 31, no. 12, pp. 1619-1626, 2011.

[41] Y. Yang, J. S. Hou, H. J. Chi et al., "Huangqi chronic obstructive pulmonary disease rat airway inflammation intervention function experimental research," Shandong Medicine, vol. 50, no. 20, pp. 49-51, 2010.

[42] Q. Li, "Research progress of pharmacological effects of Huangqi," Medicine Industry Information, vol. 3, no. 11, pp. 128129, 2006.

[43] L. Chen, B. B. Sun, T. Wang et al., "Cigarette smoke enhances $\beta$-defensin 2 expression in rat airways via nuclear factork- $\kappa \mathrm{B}$ activation," European Respiratory Journal, vol. 36, no. 3, pp. 638645,2010

[44] X. Yuan, S. Sun, S. Wang, and Y. Sun, "Effects of astragaloside IV on IFN-gamma level and prolonged airway dysfunction in a murine model of chronic asthma," Planta Medica, vol. 77, no. 4, pp. 328-333, 2011.

[45] R. Guo, M. H. Pittler, and E. Ernst, "Herbal medicines for the treatmet of COPD: a systematic review," European Respiratory Journal, vol. 28, no. 2, pp. 330-338, 2006.

[46] X. An, A. L. Zhang, A. W. Yang et al., "Oral ginseng formulae for stable chronic obstructive pulmonary disease: a systematic review," Respiratory Medicine, vol. 105, no. 2, pp. 165-176, 2011. 


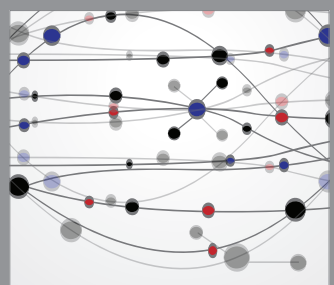

The Scientific World Journal
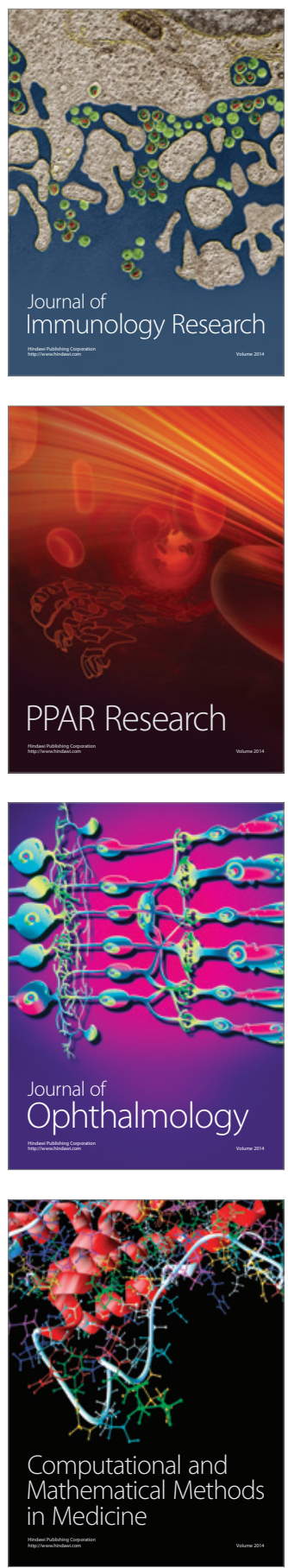

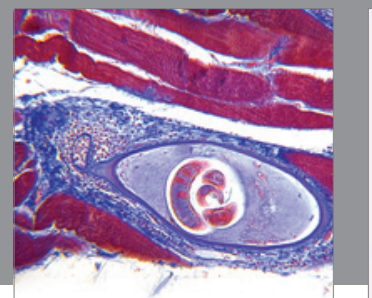

Gastroenterology

Research and Practice
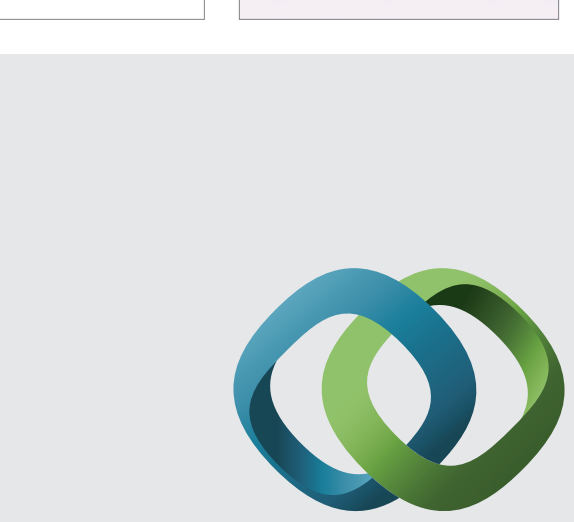

\section{Hindawi}

Submit your manuscripts at

http://www.hindawi.com
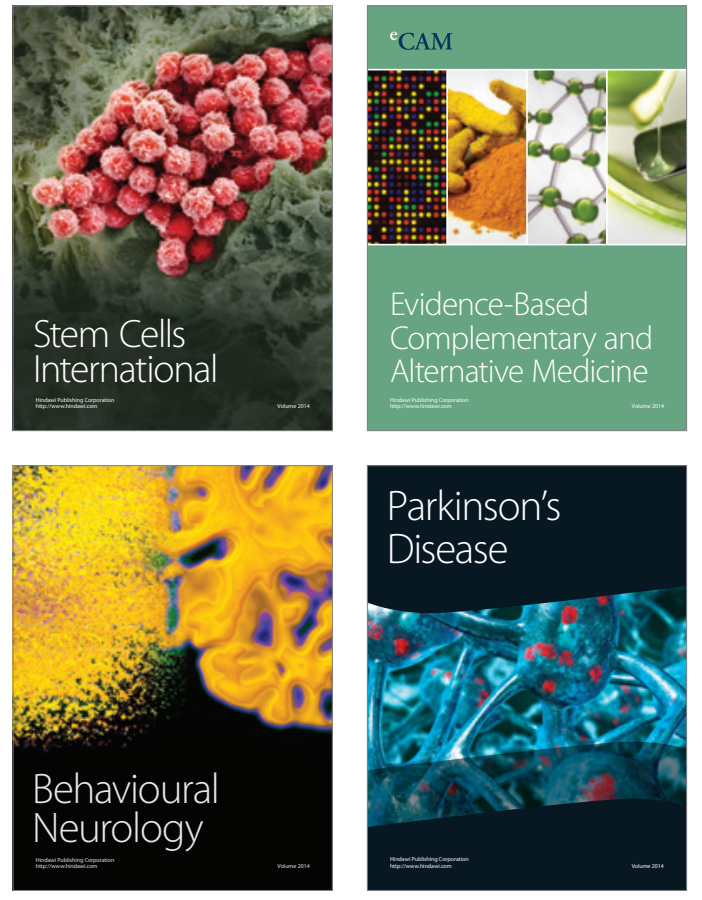
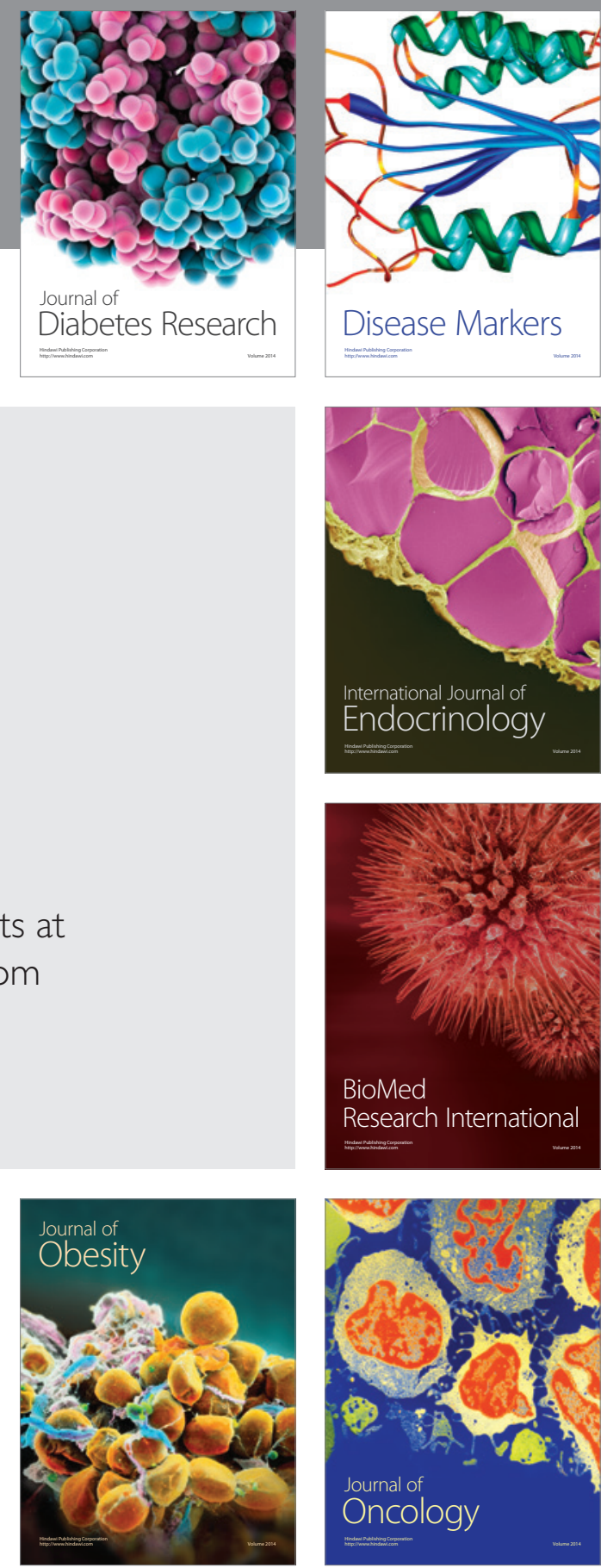

Disease Markers
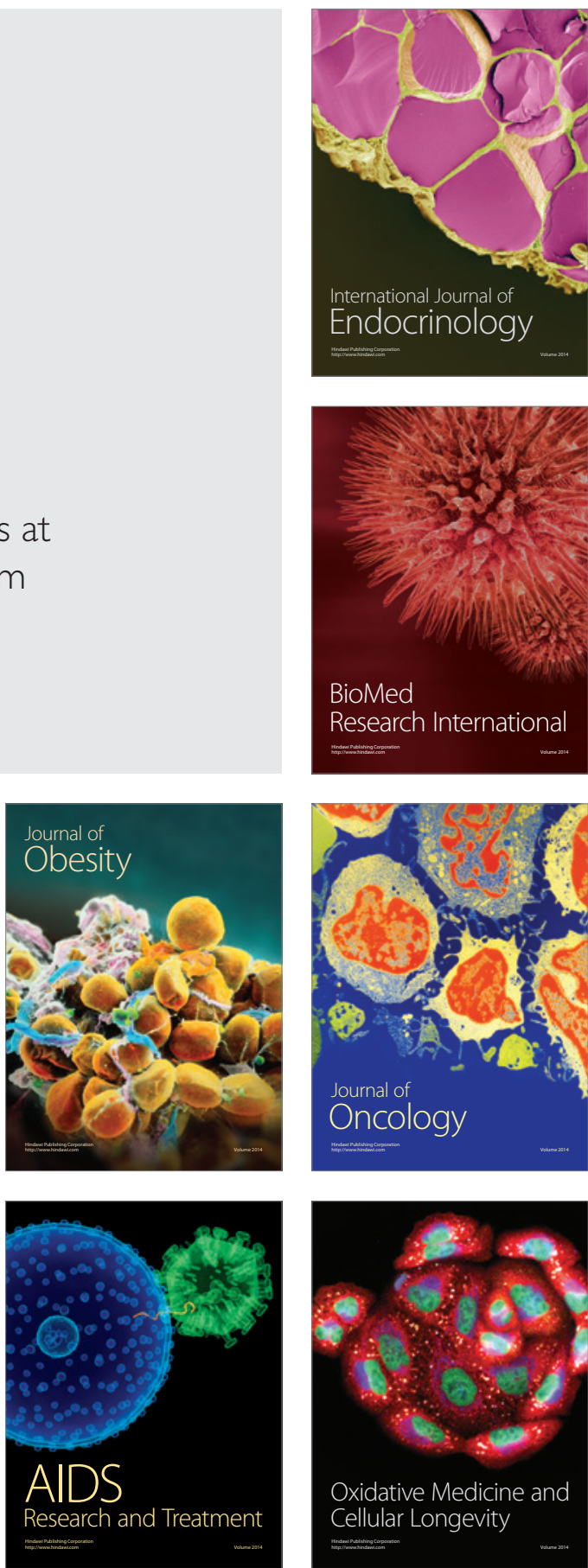